\title{
An Embedded Cloud-Based Video Surveillance System
}

\author{
Aderonke J., Ikuomola \\ Department of Mathematical Sciences, \\ Ondo State University of Science and Technology \\ Okitipupa, Nigeria \\ E-mail: deronikng@yahoo.com, aj.ikuomola@osustech.edu.ng
}

\begin{abstract}
Security has become increasingly important in recent years. Modern advances in embedded systems, electronics and communications technologies have led to the miniaturization and improvement of the performance of cameras and networking. These changes have given rise to the development of several security systems and technologies. The challenges faced by video surveillance system are namely high cost of ownership, poor accessibility and difficulty in achieving security. Traditional video surveillance demands for a huge amount of storage space to store huge amount of data coming from multiple sensors. Also, once the storage disks are damaged or destroyed, the footages will be unavailable, and thus backup is absolutely needed. In this work, an embedded cloud-based video surveillance system was designed. The embedded cloud-based video surveillance system stores footages in the cloud, eliminates unwanted capture by recording video only when motion is detected, it also aid the human operator in real-time threat detection through the message sent to the user containing video captured. With embedded cloud-based video surveillance system, the camera is activated when motion is detected and the video clips are sent to the user mail through the use of python script.
\end{abstract}

Keywords: Cloud; Embedded System; Video; Surveillance

CISDI Journal Reference Format

Ikuomola, A.J. (2019): An Embedded Cloud-Based Video Surveillance System. Computing, Information Systems, Development Informatics

\& Allied Research Journal. Vol 9 No 1.Pp 1-6. Available online at www.cisdijournal.org

DOI Affix - https://doi.org/ 10.22624/AIMS/CISDI/V10N1P1

\section{INTRODUCTION}

Video surveillance systems are widespread and common in many environments. Video surveillance has been a key component in ensuring security at airports, banks, casinos, and correctional institutions. More recently, governments' agencies, businesses, and even schools are turning toward video surveillance as a means to increase public security. These changes have given rise to the development of several security systems and technologies. Surveillance is defined as monitoring of the behaviour, other changing information, activities, observing or analyzing particular area for the purpose of influencing, directing, managing or protecting. With the proliferation of inexpensive cameras and the availability of high-speed, broad-band wireless networks, deploying a large number of cameras for security surveillance has become economically and technically feasible $[3,4]$. Traditional video surveillance demands for a huge amount of storage space to store huge amount of data coming from multiple sensors. Recording everything captured by a surveillance camera consumes the large storage space and hence limits the duration of video that can be stored. However, typical surveillance systems cannot cope with the continuous demand for massive storage. In addition, recording everything makes it time-consuming for a human to review the stored video. All these disadvantages limit the effectiveness of traditional video surveillance. 
To solve these problems, recording only crucial images that contains important information and also a cloud storage comes as a rescue that can connect different types of network storage devices to meet specific requirements of surveillance systems, such as (a) record media streams in higher frame rate without dropping frames, (b) cost-effective storage for longer retention of media data, and (c) elastic storage capability for varying demand and future growth, among others. With cloud computing, organizations can easily access their footages at any time without worrying about how to manage the storage facilities [5]. Besides, the cloud storage is considered as highly available, always-on, and more reliable than the local storage. As a result, it cannot only meet the needs for large surveillance systems, but also provide intelligent video analytic as a service on-demand to several customers [7, 2].

\section{LITERATURE REVIEW}

[7] uses a robust motion detection algorithm for real-time motion detection by considering image that contains motion in the scene. This is done with a web camera and a motion detection algorithm that detects motion. The motion detection algorithm robustly distinguishes motion from lighting changes. Web camera can take the snapshot of the moving object and at the same time, it will activate the warning system before storing the frames on the memory. [4] designed a Video Surveillance System which has video output from Charged Coupled Device (CCD) camera. This video output was divided into video sequences that was input for process called preprocessing. The system uses people tracking algorithm for recognition of moving objects on the background, head detection and luggage detection. The system prevents forbidden person from entering a secured zone or leaving suspicious luggage in a guarded room. Background detection remains a challenging problem in applications with difficult circumstances, such as changing illumination, waving trees, water, video displays, rotating fans, moving shadows, inter-reflections, camouflage, occasional changes of the true background, high traffic, etc.

[8], presented Vigil, a real-time wireless video surveillance system that leverages edge computing to enable wireless video surveillance to scale to many cameras and expand its region of coverage in a crowded wireless spectrum.

[1] presented an IP based surveillance model for a University Campus. The model developed enables video streams to be sent over the internet for remote monitoring irrespective of the access locations, it goes a long way in addressing problem associated with maintaining security, deter crime, monitoring environment and protecting lives and properties on the campus. The design architecture provides opportunity for real time viewing of video streams and opportunity to query previous recorded video feeds in terms of date and time specified but the storage medium (server) may not be sufficient for storing data after several months.

[3] designed and implemented an embedded surveillance system with a cloud storage server connected to wireless networks to detect an intruder at home/room when nobody is present. The surveillance system consists of two aspects: monitoring and control parts. For the monitoring part, an Arduino Yun board is used to be the platform using wireless networks (Wi-Fi) connecting cloud networks. For the control part, Arduino Uno development board is used to be an auxiliary platform to switch (ON/OFF) LED module and to control a servo motor (MG995) as well as the camera (Logitech C310). For a purpose of remote control, the platform combines with a Bluetooth kit (HC-06). Mobile phone users can easily use a simple self-developed APP to control these devices. The main challenge is that Bluetooth limits the effort of remote control aspect as the distance between the system and mobile phone is limited.

[6], proposed an Automated Exam Invigilation (AEI) system called Frame Subtraction to keep track of the movements in exam hall. Whenever any movement of persons in the exam hall is detected, the system sends a notification. The image processing tools in MATLAB are used to recognize the face by comparing the images stored in the database and the images captured by CCTV camera. The problem is that a little expression or accessory can change the whole look of a person. Presence of spectacles, mustaches, beard, surgery on face, aging and pimples can lead to unnecessary dismissal of the same face. This is called FACE REJECT. 


\section{ARCHITECTURE OF AN EMBEDDED CLOUD-BASED VIDEO SURVEILLANCE SYSTEM}

The architecture of an embedded cloud-based video surveillance system is presented in figure 1. The system comprises of the following components: Raspberry pi, Power Supply, Webcam, Internet, cloud storage and user interface. Raspberry pi 3 is the main structure of the surveillance system and it is a platform using wireless networks (Wi-Fi) to connect to the cloud network. The Raspberry pi motion daemon software is a program that monitors the video signal from one or more cameras and as soon as motion is detected, the raspberry pi starts to record the video and also takes pictures at random intervals. The picture and video taking is sent to specified cloud storage, goggle mail in this case. The motion detection also triggers a Python script which was created and sends a notification to the user's email address attached in the script about the intruder/motion detected. After receiving the emailed message, users have options to monitor the situation via livestreamed Webcam or other preventive measures.

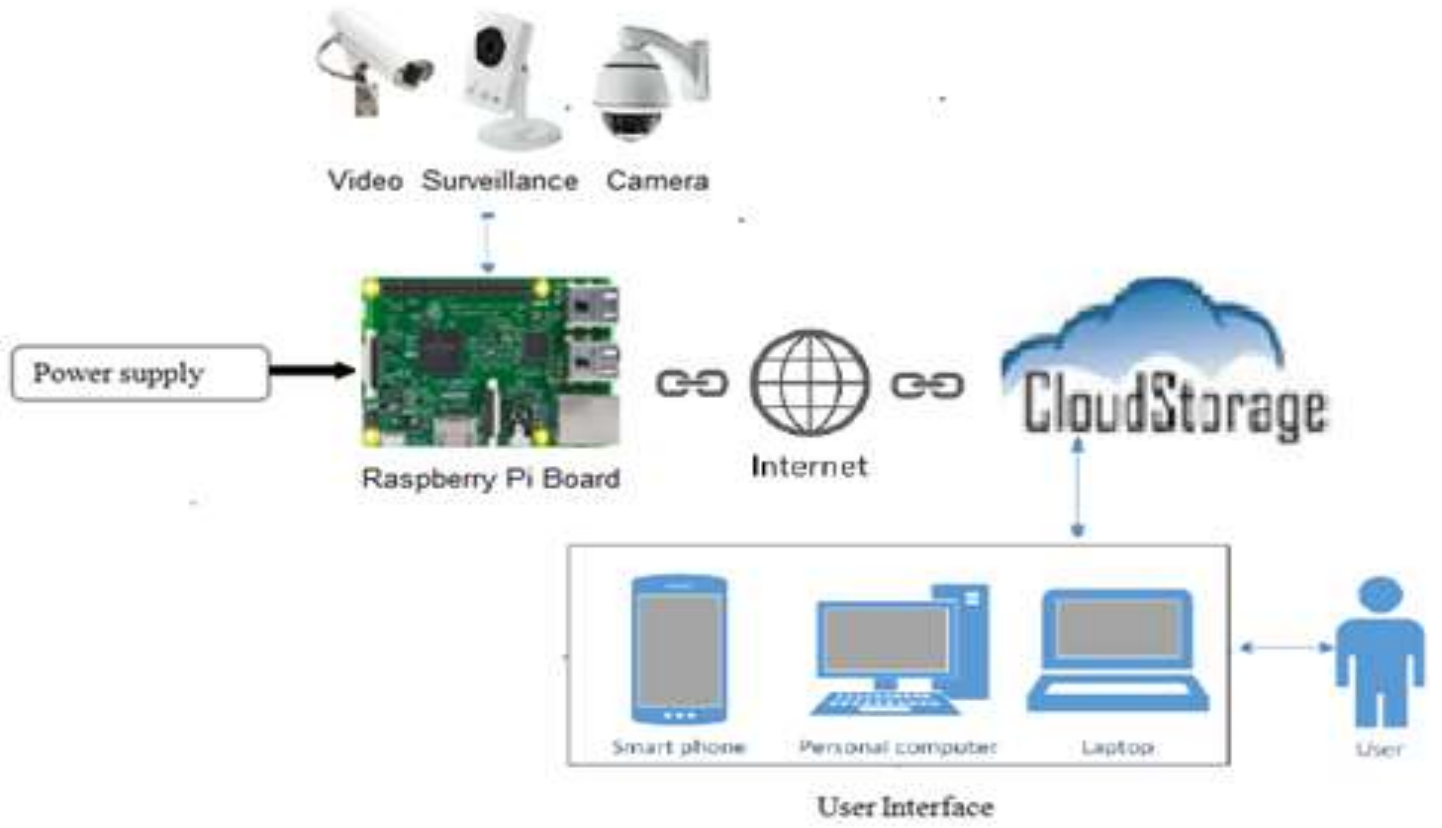

Figure 1: An embedded cloud-based video surveillance system architecture

\subsection{Component of the Design}

User Interface: This allows the user to interact with the system. User interface could be smartphone for viewing video from mail or computer system for livestreaming.

Raspberry pi 3 (Microprocessor): It is a series of small single-board computers. The software is heavily based on the Raspberry pi Motion Daemon Software. The motion daemon software is a program that monitors the video signal from one or more cameras and is able to detect if a significant part of the picture has changed or in other words, it can detect motion. As soon as motion is detected, the raspberry pi starts recording video and taking pictures at random intervals. The picture and video taking is sent to specified cloud storage, goggle mail in this case. The motion detection also triggers a Python script which was created to sends a notification about the intruder to the user's email address attached in the script. 
Power Supply: The raspberry pi, requires a 2A, 5V power supply system. Although the amount of current drawn depends on the other appliances connected to the pi, a current rating of between 2-2.5 amps is safe for use with most components.

Camera : This is used for video and picture acquisition. HV-N5086 Havit USB camera was used due to the balance between cost and performance. The camera used had lights attached to it which can be used for night vision.

Internet: is a means of connecting systems globally. To connect the raspberry pi to the internet, an Ethernet cable was used to connect it to a computer system with Wi-Fi connection.

Cloud Storage: Could be iCloud Drive, Google Drive, One Drive or Dropbox. The cloud storage for storing video used for the system is Google Drive. Google Drive is like Dropbox. It creates a folder on the computer, where files can be stored, and apps on mobile devices lets the contents of the folder to be stored. Individual Google apps, such as Docs, Sheets, and Slides, store their files on Google Drive as well. Google Drive can be used on Mac and Windows, and on Android or iOS devices. Google Drive can also be used with a Chromebook, or through a web browser. With 15GB free, Google makes storage comfortable. It can store as many as possible, and if more storage is needed, Google provides more storage.

\section{IMPLEMENTATION AND RESULT}

It was implemented using raspberry pi motion daemon, python scripts and putty application to send videos captured by the camera to a specified google mail account. Figure 2 shows the Screenshot of motion setting environment.

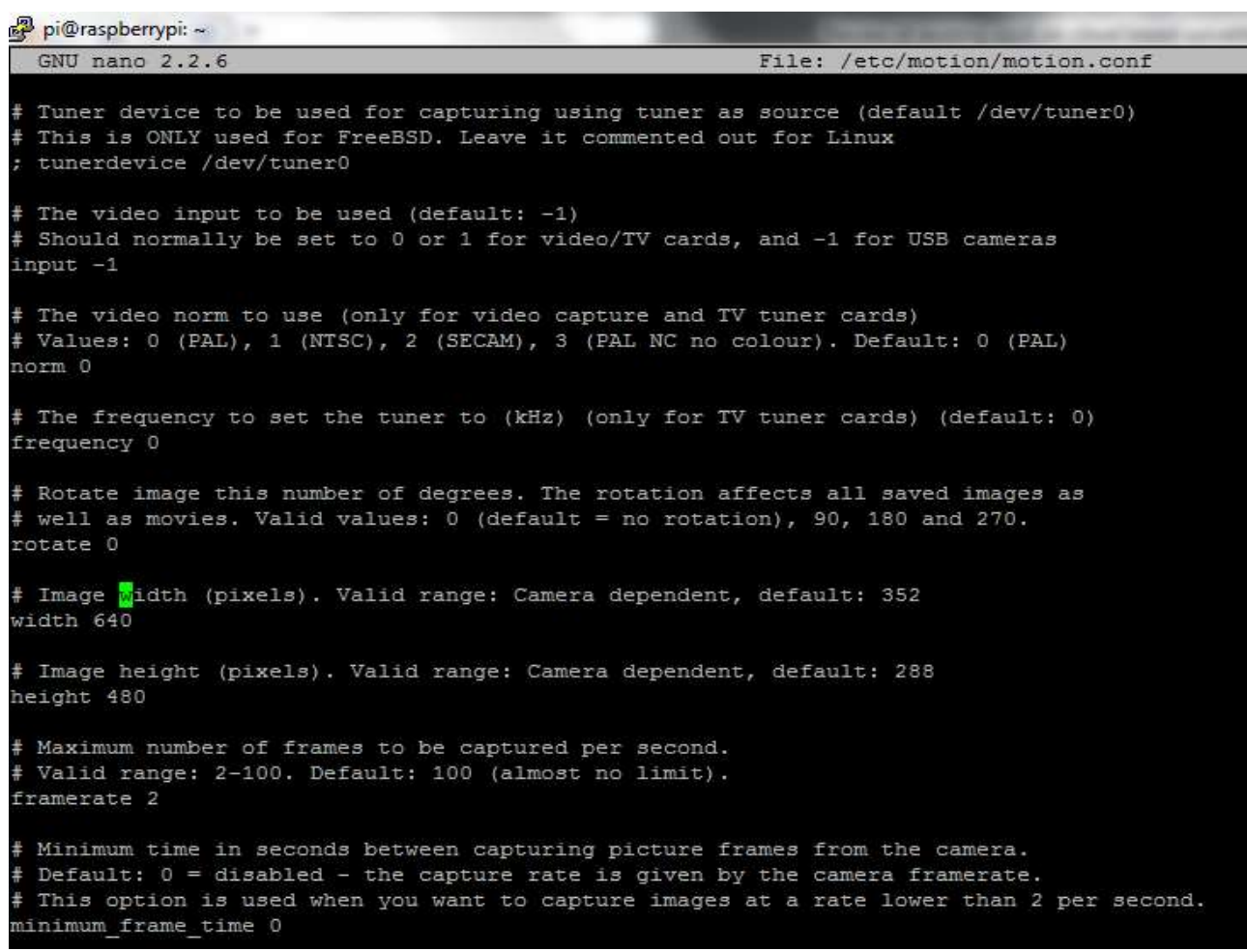

Figure 2: Screenshot of motion setting environment 


\subsection{Preliminary Testing of a Cloud-Based Video Surveillance System}

This section describes how the video surveillance system was created and tested. SSMTP was the application used to send the mail to the mail server. SSMTP is a highly simplified mail transfer agent (MTA) that is used when a system administrator needs to send mail from a server, but doesn't need to set it up as a fully-fledged mail domain. Since it uses SSMTP, it is necessary to enter the email address and password of the account the notifications will be sent to. The content text can also be adjusted within the program. There's a native library in Python to send emails: $\underline{s m t p l i b}$. In this specific script, when a motion/intruder is detected, the user is alerted and the system sends the screenshots in figure 4 to Google Drive (Gmail) in figure 6 which is a predefined cloud storage system. Figure 3 shows the image of cloud based video surveillance system while figure 4 shows the motion alert from the video surveillance system to the user email.

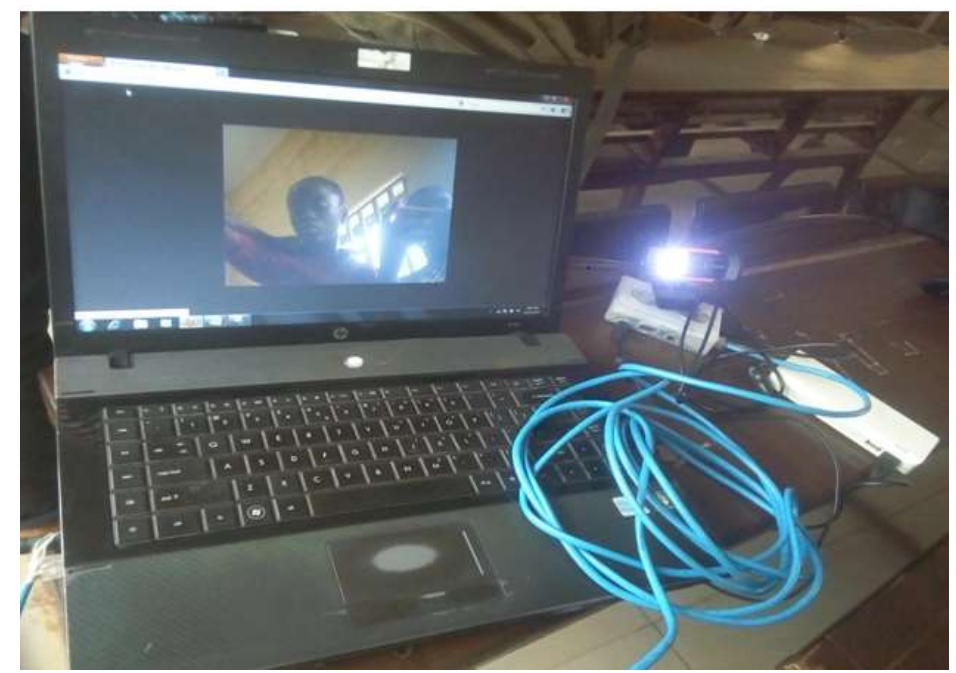

Figure 3: Image of cloud based video surveillance system

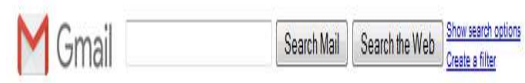

\begin{tabular}{|c|c|c|c|c|}
\hline Composilllail & Active & Reporifopan Delese & Nurekcions.. - Go Retiest & 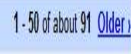 \\
\hline Ihbox 27999 & [ me & & 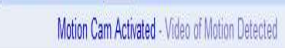 & ONa26 \\
\hline 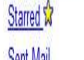 & [ $\mathrm{me}$ & & 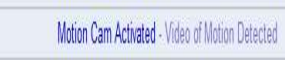 & Alow2 \\
\hline 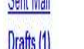 & [ $\mathrm{me}$ & & 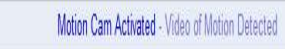 & Alow25 \\
\hline All lla & I $\mathrm{me}$ & & 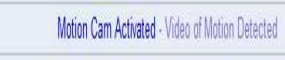 & Alow25 \\
\hline Span || $1 \mid$ & I $m$ & & 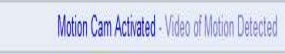 & Alows \\
\hline Trash & I me & & 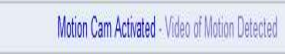 & Alow25 \\
\hline Contacts & I me & & 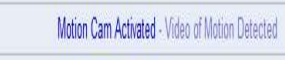 & Alow25 \\
\hline Latele & [ me & & 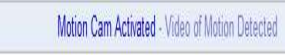 & Alow5 \\
\hline
\end{tabular}

Figure 4: Motion alert from video surveillance system to the user email 


\section{CONCLUSION}

Users were once satisfied with traditional surveillance which involves storing footages on storage disks which when damaged or destroyed, the footages will be unavailable and also keeping an eye on the system all day long to detect intruder. From the perspective of real-time threat detection, it is well known that human visual attention drops below acceptance levels, even when trained personnel are assigned to the task of visual monitoring. In this research work, an embedded cloud based video surveillance was designed. The system is a surveillance system used for monitoring an area of interest and to detect motion.

With embedded cloud based video surveillance system, the camera is activated when motion is detected and the video clips are sent to the user Gmail through the use of python script. The embedded cloud based video surveillance system stores footages in the cloud, eliminates unwanted capture by recording video only when motion is detected, it also aid the human operator in real-time threat detection through the message sent to the user containing video captured.

In future, it might be necessary to live stream the event on other devices such as mobile phones and also to use a wireless adapter thereby increasing the dependency on the user network connection.

\section{REFERENCES}

[1] Akanbi C. . and Eludire A., (2015). A Mobile Access IP-Based Video Surveillance Model for a University Campus Security. African Journal of Computing \& ICT, 8 (4), 181-184.

[2] Hossain M. A. (2014). Framework for a Cloud-based multi-media surveillance system. International Journal of Distributed Sensor Network.

[3] Hsueh J., Huang Y., Dong B. and Lee C.(2017). An Embedded Cloud Surveillance System Design. Department of Electrical Engineering, I-Shou University, Kaohsiung City, Taiwan.

[4] Ovsenik L. Kazimirova A., and Turan J. (2010). Video Surveillance Systems. Acta Electrotechnica et Informatica, 10 (4), 46-53

[5] Song B., Hassan M.M., TianY., Hassan M. S. and Alamri A. (2015). Remote display solution for video surveillance in multi-media cloud. Multimedia Tools and Applications. 1- 22.

[6] Shenbagam P., Aravinth K., ChakravarthyJ., and Kumar R. (2017). Exam Hall Invigilation Using CCTV Camera. SSRG International Journal of Computer Science and Engineering, 64-67.

[7] Suradkar H, Kolte A., Jamdade S., and Gokhale S. (2015). Automatic Surveillance using Motion Detection. International Journal of Engineering Research and General Science, 3 (2), 525-529.

[8] Zhang T., Chowdhery A., Bahl P.,Banerjee S., and Jamieson K. (2015). The Design and Implementation of a Wireless Video Surveillance System. MobiCom ' 15 proceedings of the $21^{\text {st }}$ annual international conference on mobile computing and networking. 426-438. 